Journal of Computer Science 8 (4): 591-598, 2012

ISSN 1549-3636

(C) 2012 Science Publications

\title{
Customer-Made 3×3 Polymer Optical Fiber Splitter Based on Green Technology using Fusion Technique
}

\author{
Mohammad Syuhaimi Ab-Rahman, Nik Nur Syahirah Mohammad, \\ Hadi Guna, Mohd Hazwan Harun and L.S. Supian \\ Department of Electrical, Electronics and System Engineering, \\ Faculty of Engineering and Built Environmental, \\ Institute of Space Science (ANGKASA), \\ University Kebangsaan Malaysia 43600 UKM Bangi, Selangor, Malaysia
}

\begin{abstract}
Problem statement: Polymer Optical Fiber (POF) has many advantages making it the choice of today's communication, especially in automotive industry. This communication requires low cost ways for much information to be sent simultaneously. Because of this, using Wavelength Division Multiplexing (WDM) concept, a low cost and green $3 \times 3$ POF splitter is fabricated. It is fabricated using cheap and easy-to-find tools such as metal tube and candle. Approach: The fabrication is being done by taking three strands of POF and heating the middle parts so that it will melted and fused together until it elongated and shrink to a diameter of $1 \mathrm{~mm}$. Successfully fabricated POF splitter are characterized by measuring the output losses, before and after the POF's end is being smoothed and polished using two sand study of different degree of coarseness. This is to observe the effect of polishing on optical signal losses. POF splitter's losses are also measured on different temperature to observe the effect of temperature on the splitter's performance. Results: Based on the result, it is obvious that cleanliness and a flat POF's end surface influenced the losses with a reduction between 0.3- 5.0 dB. Temperature, even though subtle, does also affect the signal losses with increased losses averaging around $0.3 \mathrm{~dB}$. Overall, the losses in the fabricated POF splitter are still high, but show promising improvement with amelioration of fusion technique and the use of tools with higher quality. Conclusion: This low cost fabrication technique is believed to commercially produce cheap POF splitter, but with more in-depth research on the fusion technique, source of losses and ways to minimize the losses and improve output optic signals.
\end{abstract}

Key words: Polymer Optical Fiber (POF), Wavelength Division Multiplexing (WDM), Poly-Methyl Methacrylate (PMMA), Numerical Aperture (NA), Immune to Electromagnetic Noise (EMI)

\section{INTRODUCTION}

Copper wire is a conventional conductor that used for data transmission from one place to another place. However, the use of copper wire has many shortcomings such as the transmitted signal will be affected by electrical characteristics such as resistance and interference that resulting during the data transmission and dependence on transmitted distance. This means, the further distance of transmitted signal, the decreasing signal strength will be (more likely to lose data). In addition, the resistance in copper will delayed the current flow or signal and it will cause a lower transmission rate. Not only that, the interference and noise can also occur in data transmission and cause the copper wire to be into one of unsuitable transmission medium.

In addition to the use of copper wire, optical fiber glass is also used as a medium of data transmission. But it's characteristic of having a small diameter cause the optical fiber glass to be not flexible. In addition, the installation of optical fiber glass requires a special handling and care, thus resulting in high installation cost. However, it can be used for long distance data transmission and it is not suitable for use in data transmission of a vehicle. To increase the rate of data transmission, Wavelength Division Multiplexing (WDM) technique is used. WDM is a technique in which some amount of optical signal is transmitted simultaneously on one optical fiber by using different

Corresponding Author: Mohammad Syuhaimi, Department of Electrical, Electronics and System Engineering, Faculty of Engineering and Built Environmental, Institute of Space Science (ANGKASA), University Kebangsaan Malaysia 43600 UKM Bangi, Selangor, Malaysia 
wavelengths. Because of different wavelengths, they will not interfere with each other and this will cause the bandwidth to be higher.

The main objective of this study is to fabricate $3 \times 3$ POF splitter with low cost and green technologically. A successful fabricated POF splitter will be detailed in order to see its performance. To achieve this objective, several tasks will be done such as:

- The fabricated POF splitter has to use a low cost fusion technique by using tools that are already available. Fusion technique is also easy to perform without having to learn a lot of knowledge about the operation

- The fabricated POF splitter will go through a process of characterization. This process is carried out to see the performance of fabricated POF splitter. Amongst the parameters that need to be studied is excess loss, insertion loss and separation ratio

- The fabricated POF splitter will also go through on temperature experimental. This process is carried out to see the performance of POF splitter at different temperatures. Also, to determine the effect of different temperature on POF that can be applied at various environments with high temperature

Optical fiber is a type of transparent fiber that allow light through it from one end to another. It allows this optical fiber to be a kind of wavelength which upholds the principles of full internal reflection. Data will be converted into light before being transmitted to optical fiber that will reflect until the end of the fiber. Since the optical fiber which allow a long distance communication in higher bandwidth compared to other forms of communication, the use of optical fibers are now more widespread. In general, optical fiber consisting of core that has high transparency as shown in Fig. 1. This core will be covered with a coating of lower refractive index so that the principle of full internal reflection can be fulfilled. In addition, optical fiber will be coated with another layer which is jacket layer; where it can protect the optical fiber from heat and humidity. Also, it can protect the optical fiber from damage and disrupt the propagation of light.

POF is an optical fiber based on polymer material where the core layer is made from PolyMethyl Methacrylate (PMMA) and the coating layer is made from perfluoroplastic (Ab-Rahman et al., 2010a). In large diameter fiber, the core consists of $96 \%$ on the cross-section, where it allows light to go through (O'Gorman et al., 2010; Appajaiah, 2004). Familiarity, POF has a core diameter of about $1 \mathrm{~mm}$ and has a Numerical Aperture (NA) of 0.5.

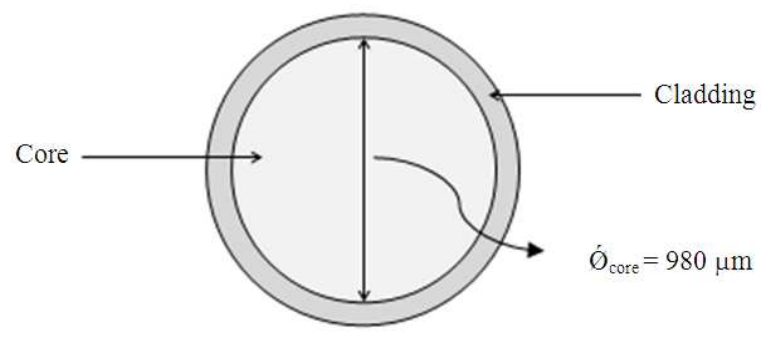

Fig. 1: Structure of POF cross section

The uses of POF in respect of communication application are now more widely (Zieamann et al., 2008). This is due to the characteristics and advantages such as flexibility, light weight, easy handling, low cost for large core diameter (Kuzyk, 2007), structure based on polymer material and simple installation (Im et al., 2002). Furthermore, POF also resistant at high temperature, Immune to Electromagnetic Noise (EMI) because of its layer made from perfluoroplastic, suitable for data communication up to $100 \mathrm{~m}$ away, high data transfer speed (400 Mbps for SI and $1 \mathrm{Gbps}$ for GI), high bandwidth (above $4 \mathrm{Ghz}$ ) and resistant to vibration (Appajaiah, 2004; Grzemba, 2008; Kuzyk, 2007; Zieamann et al., 2008). POF splitter is a type of device that split the optical power which carried by single fiber input to multiple fiber output. For multiple input in which the combined inputs make it as a single output, is called as coupling. Optical splitter using tapered fusion techniques (Fused Taper Optical Splitter) is an optical splitter based on star coupler (Ab-Rahman et al., 2009b). Normally, optical splitter that using this technique will applied a heat during the process of fusion. Optical fiber splitter is capable of functioning in two ways.

\section{MATERIALS AND METHODS}

This study will focus on the fabrication and characterization of a device prototype. Fabrication of optical splitter involves three strand of polymer optical fiber that is fusible to be a $3 \times 3$ optical splitter. Splitter fabrication process will be through four steps as shown in Fig. 2 (Ab-Rahman et al., 2009a). For fabrication of POF splitter, Fused Biconical Taper technique will be used. This technique will use all the tools available, such as candle for heating and metal tubes to avoid POF contacted directly with heat from candle Fig. 3 and 4 (Rahman et al., 2012). Only two tools involved and its low cost. In addition, this fusion technique is easy to learn without requiring special knowledge to operate. 
Fabrication method for $3 \times 3$ POF splitter needs to be done in two steps which are spinning process and POF fusion process (Harun et al., 2011). Spinning process is a process of twisting three POF strands tightly and compact on the fusible POF. Three strands of POF that have been included in metal tube is heated and twisted at the same time. Both ends of POF will be pulled and twisted in different directions at one end in a clockwise direction, while the other end will be twisted in direction of counter-clockwise with the same hand strength. At the same time, both ends of POF are also pulled towards the outer metal tube with same force (Ab-Rahman et al., 2010b). The spinning process is gradually made until it becomes neat and closely segmentation at the middle part of POF.

The next process is a process of fusion. Fusion means to include the three POF together so that they will be elongated and narrowed into a POF with a diameter of $1 \mathrm{~mm}$. This process uses the same steps as the heating process, but with the stronger twisting and pulling forces until POF is narrowed, elongated and fused; then the diameter of POF will be same as a size of original POF which is $1 \mathrm{~mm}$. Monitoring process should always be done to prevent a part of fused POF become too small and delicate, so that it cannot broken or being cut off.

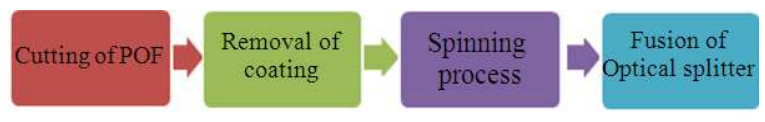

Fig. 2: Flow chart of optical splitter fabrication

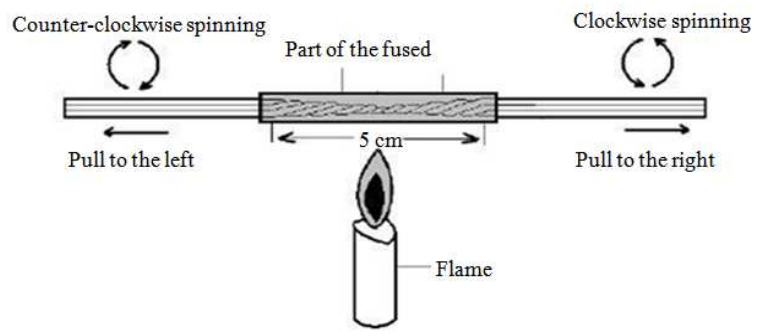

Fig. 3: Fusion processes that has been narrowed Source: Ab-Rahman et al. (2010a)

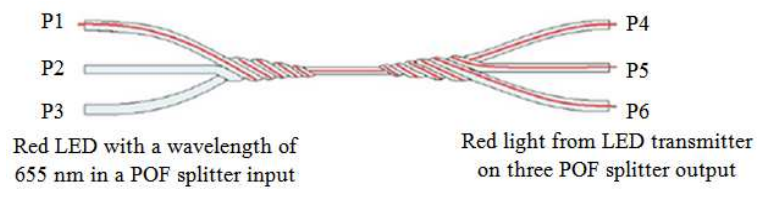

Fig. 4: Method on measurement of optical signal output Source: Ab-Rahman et al. (2010a)
After that, a characterization will be made to see the performance on this splitter. In order to measure the optical output power, LED transmitter that emits red light at $665 \mathrm{~nm}$ at one input and power meter will be used. This process is carried out for all input whether for forward bias or reverse bias. This characterization will also be repeated after the end of POF splitter surface is flattened and polished with sand study by using two different degrees of harshness. The next experiment that needs to be conducted on POF splitter is experimental temperature. POF splitter will be placed on a digital hot plate which it will be heated gradually at every increase of $5^{\circ} \mathrm{C}$ for each reading. Readings will be taken at the lowest temperature of hot plate which is at $35^{\circ} \mathrm{C}$ and it will continue until the maximum temperature that can be received by POF splitter before it melted and broken. The obtained value will be recorded in the table that has been prepared to facilitate the calculation and analysis.

\section{RESULTS}

As a result of the fabrication process, seven prototype samples had successfully fabricated. These seven samples will be through the characterization process of before and after polished. After all characterization and calculations have been made, then the graphs below are generated. Figure 5, shows a graph of excess loss, respectively for reverse bias.

The other loss that has been measured is insertion loss. Insertion loss refers to loss caused by the presence of one component in optical fiber. Figure 6 and 7, shows the insertion loss for forward and reverse bias, respectively. The value of this insertion loss has an average around 14-16 dB and it considered larger compared to POF loss which is about $1 \mathrm{~dB} \mathrm{~m}^{-1}$. Figure 8 and 9 show the separation ratio for forward and reverse bias respectively, where the yellow line refers to an ideal line. From graph, it can be seen that the ratio is deviated from an ideal separation ratio.

From a straight line drawn in Fig. 10, it can be seen that the slope of this graph is at 0.257 and 0.248 ; respectively for forward and reverse bias. This indicates that average loss increase by approximately $25 \%$ for an increasing at temperature up to $105^{\circ} \mathrm{C}$. 


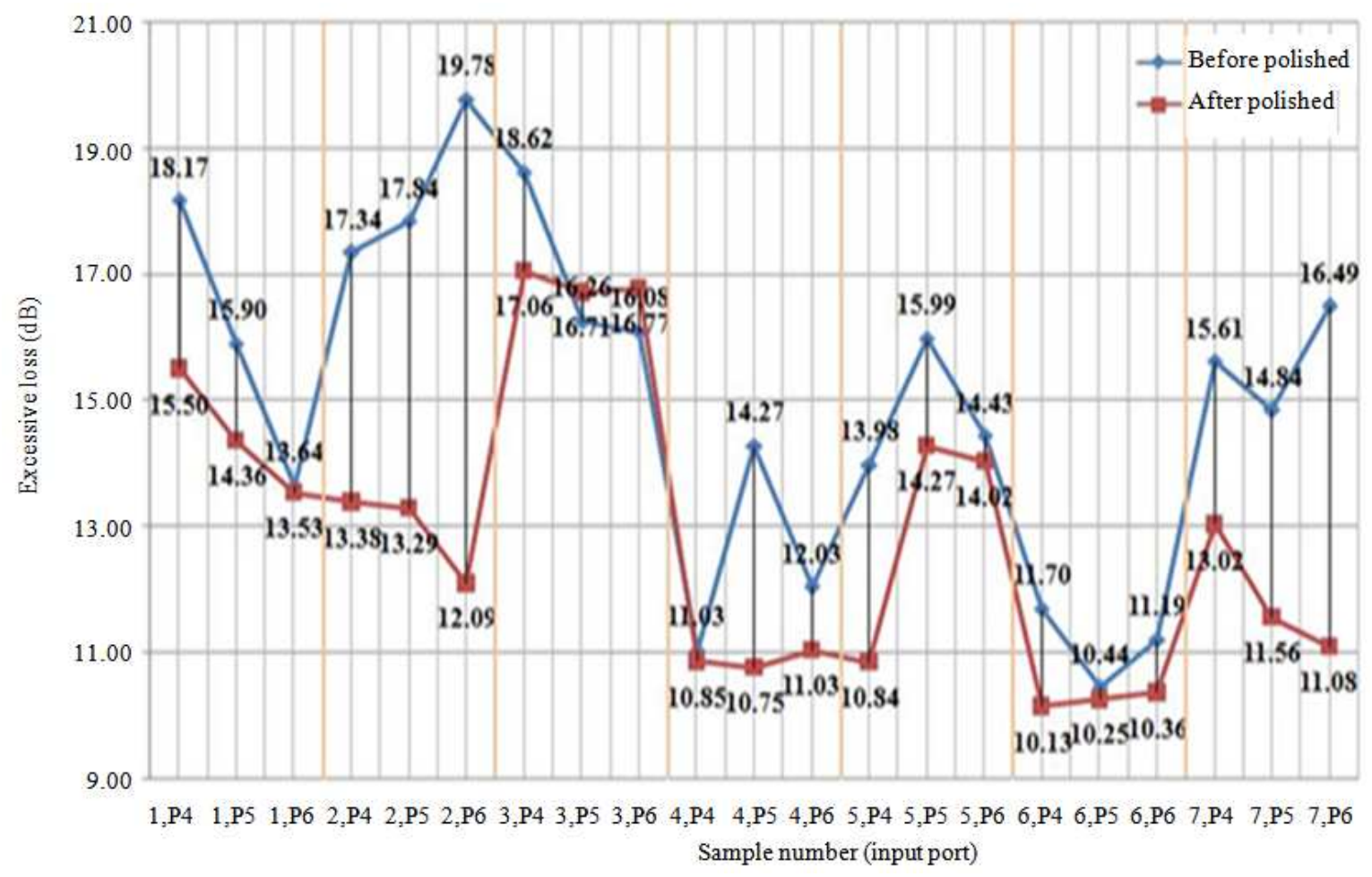

Fig. 5: Excessive loss at reverse bias

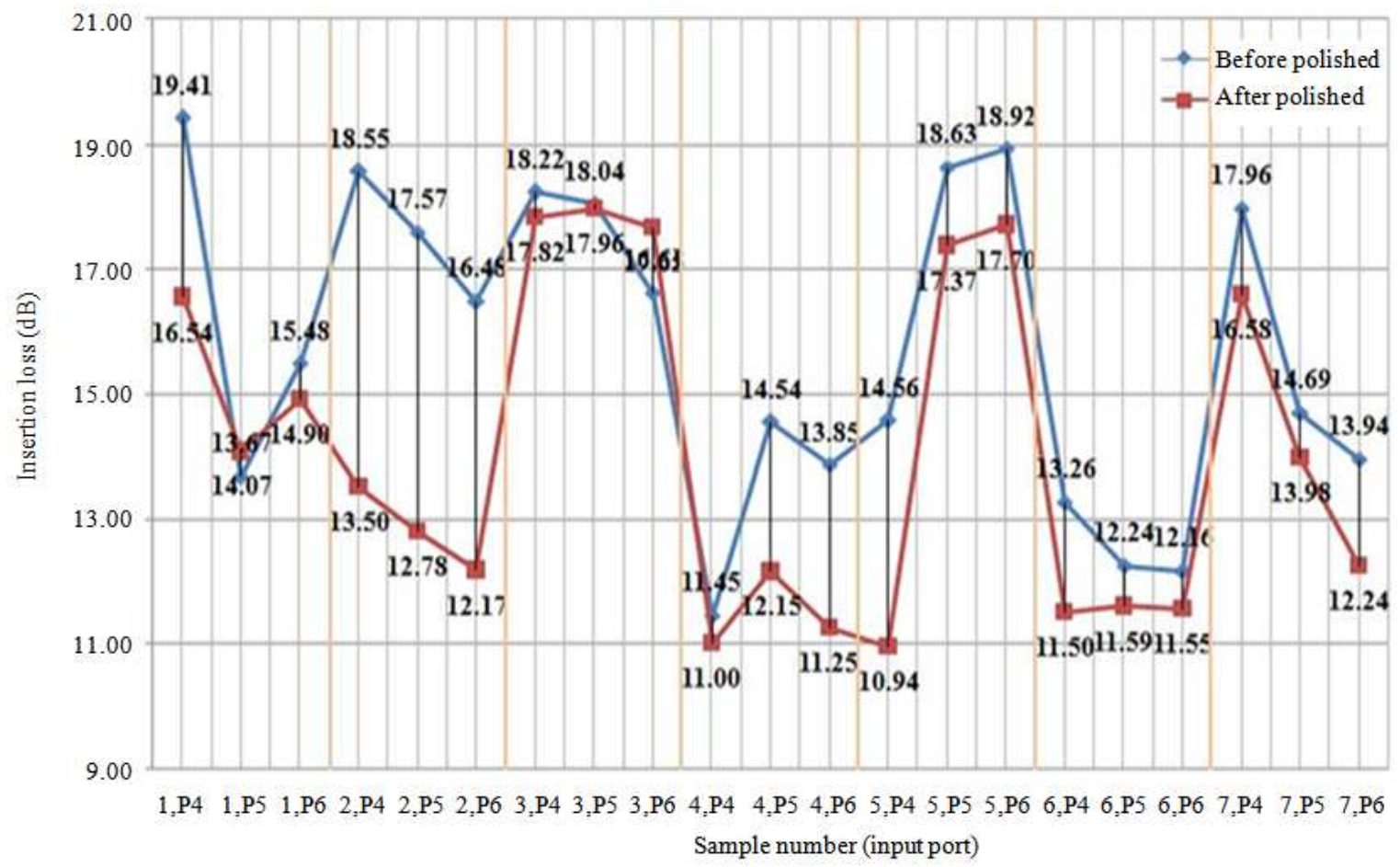

Fig. 6: Insertion loss at forward bias 


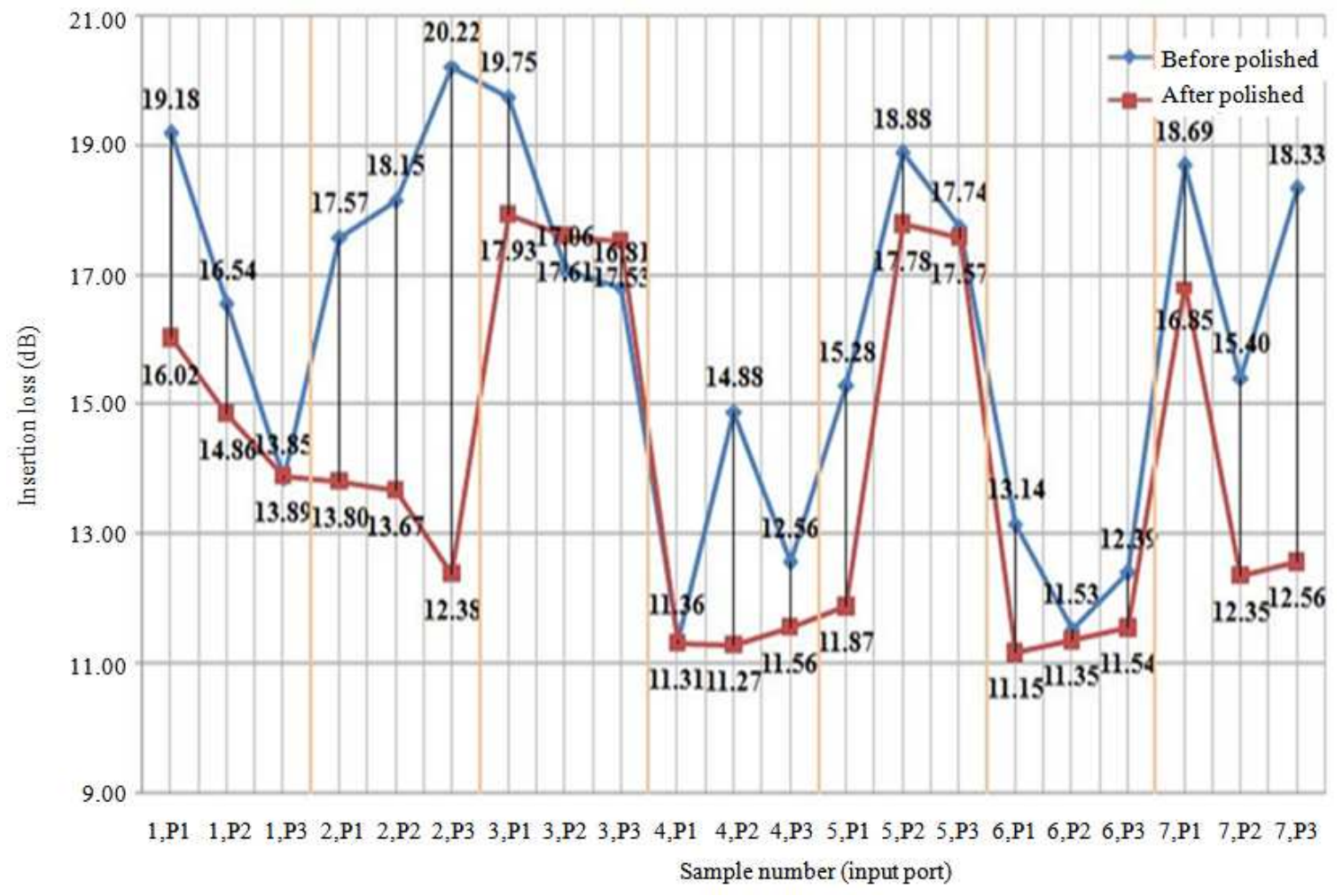

Fig. 7: Insertion loss for reverse bias

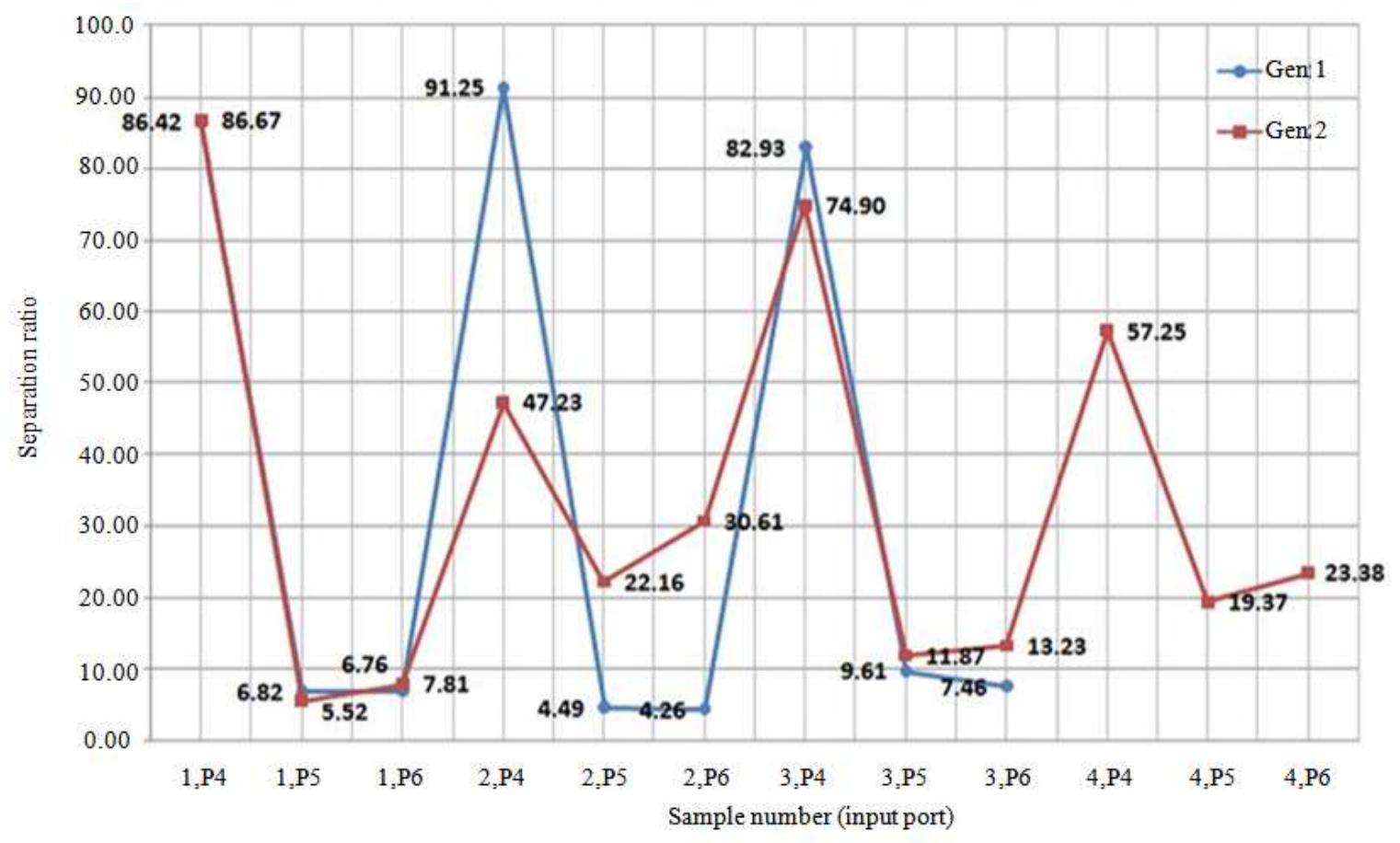

Fig. 8: Separation ratio for forward bias 
J. Computer Sci., 8 (4): 591-598, 2012

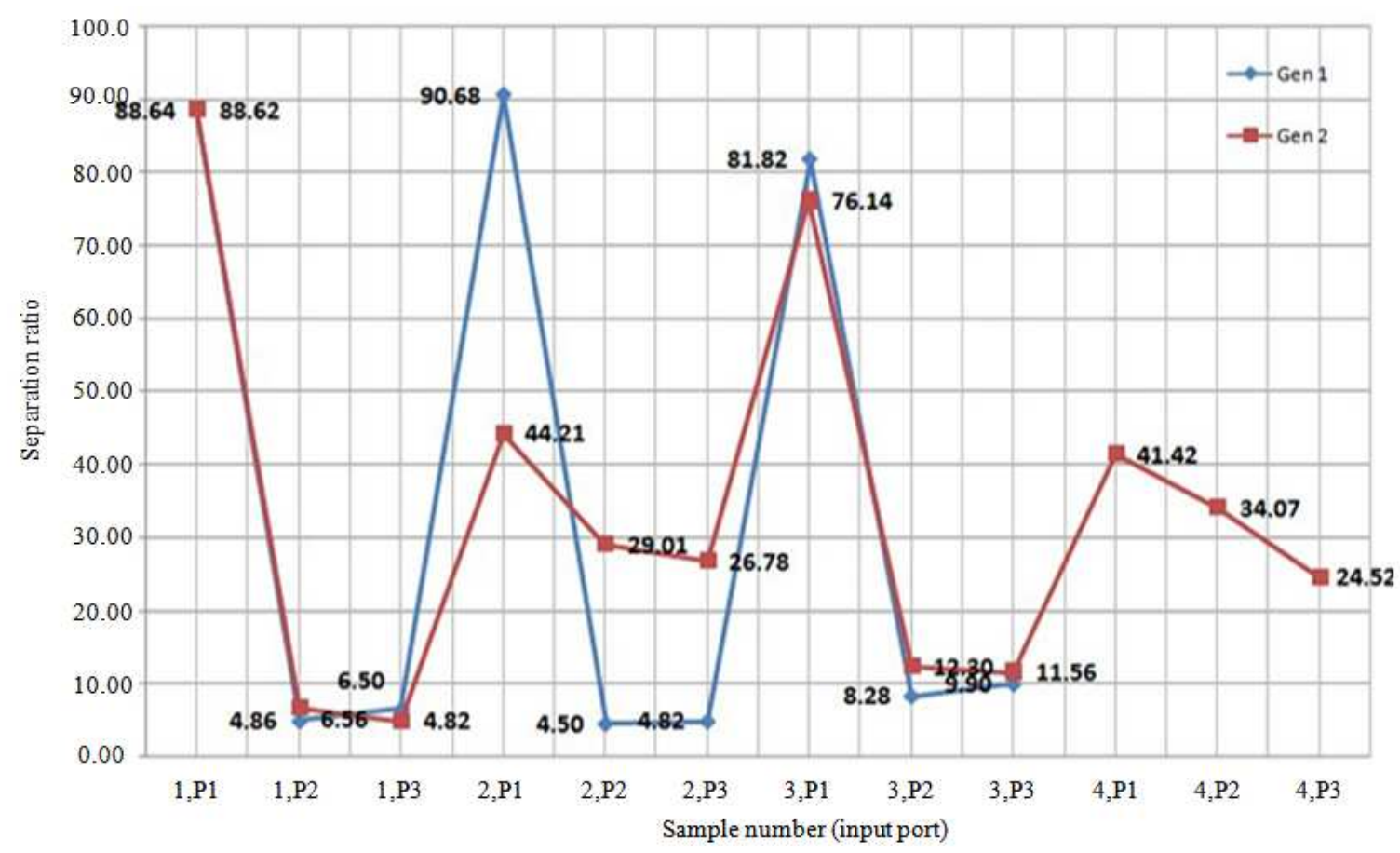

Fig. 9: Separation ratio for reverse bias

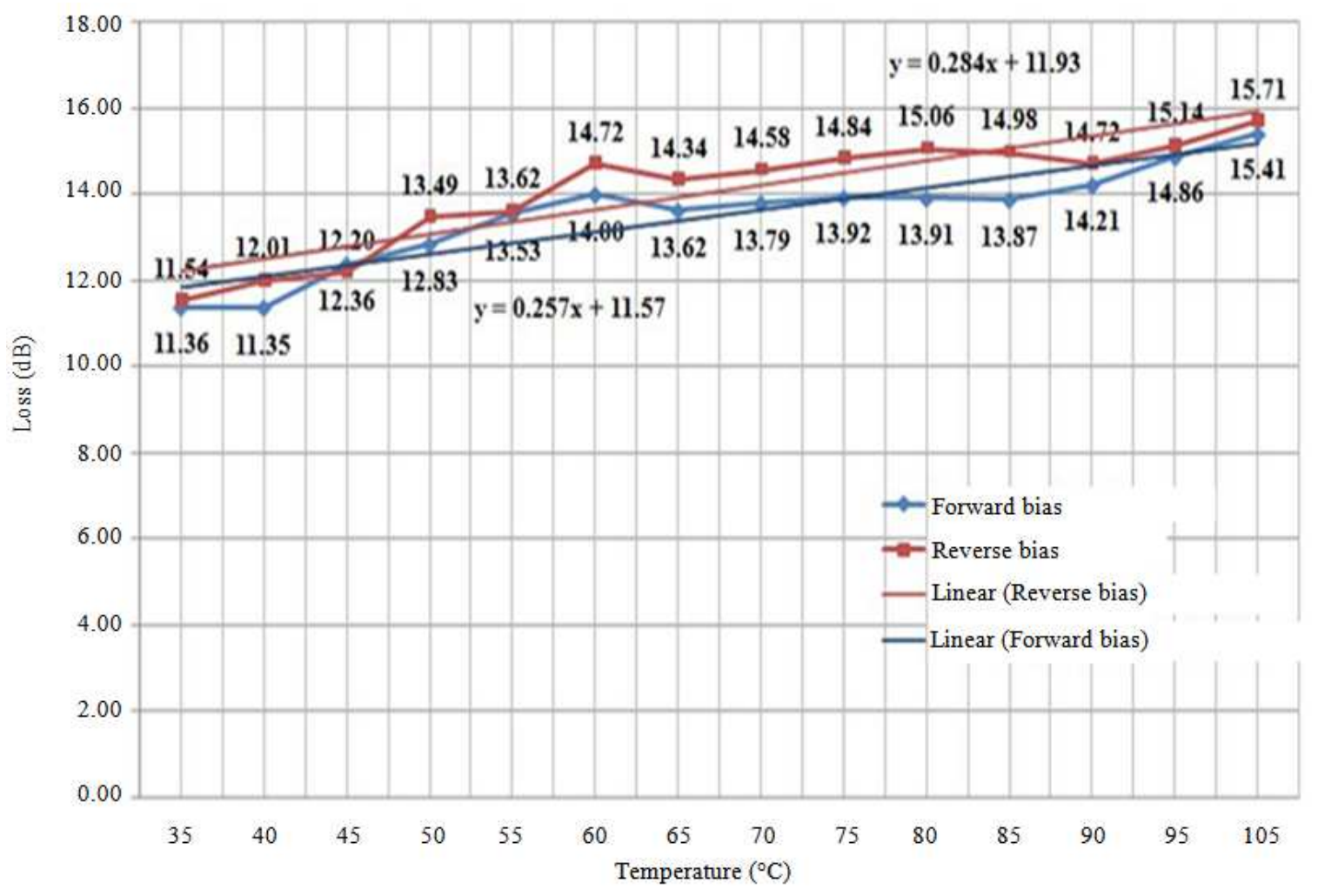

Fig. 10: Graph of temperature test 


\section{DISCUSSION}

Referring to the graph in Fig. 5 and, it can be seen that the excessive loss is high enough where most of the loss exceeds $10 \mathrm{~dB}$. It can be considered as a great loss for a communication system in which the normal loss of POF is about $1 \mathrm{~dB} \mathrm{~m}^{-1}$. This excessive loss caused by light through the POF splitter that is not refracted out entirely. This may be due to structural changes during the POF fabrication. Irregular heating during POF fabrication process and characteristic of POF that has low melting point will cause POF to be very sensitive to the heating process. Fabrication method such as pulling and twisting in excessive way can also cause this loss occurs.

By referring to Fig. 6 and 7, Loss difference between Generation 1 (sample 1-3) and Generation 2 (sample 4-7) has a significant difference. This may be due to the fusion process in which the basic structure of POF core has dramatically changed, thus it will cause a non-perfect structure. POF narrowing diameter has led to the changes in its optical characterization including on numerical aperture value and critical angle of direct light.

Besides that, the pulling and spun force during the fusion process also plays an important role in loss impact. Too much force will cause the POF core structure to be damaged and high in loss. In the other hand, too little force also will cause the three strands of POF to be not properly spun and fusion thus it may result in loss. Other than loss, separation ratio of POF splitter is also being calculated. Ideally, the separation ratio is $33: 33: 33$ in which the optical signal light has split uniformly for each output.

In Fig. 8 and 9, It shows that the fusion and twisting process has not fulfilled the criteria for POF splitter in order to do an efficient splitting. POF splitter is only partially fused, thus it caused the light cannot be uniformly split for each output.

One of the important experiments is on temperature. In automotive field, there is a need on POF splitter that can supports on high temperature. Thus, an experiment is carried out to see the POF splitter performance at different temperature. The resulting graph shows a loss on both forward and reverse bias; where a straight line is drawn in order to look at the slope of graph. Based on the graph, there was an increase in loss when the temperature is increasing. However, the increasing value is still small even at the maximum allowable temperature for POF $\left(85^{\circ} \mathrm{C}\right)$. POF splitter shows not much difference in loss even at temperature above $85^{\circ} \mathrm{C}$ until it reached $105^{\circ} \mathrm{C}$. In Fig. 10, We can see that the POF splitter can still be used up to a temperature of $100^{\circ} \mathrm{C}$. Furthermore, its jacket has been removed and it will cause the POF splitter to be less resistant to high temperature. If the POF splitter still has a jacket that functioned as its core structure, probably it is able to accommodate with higher temperature. From this experiment, the final objective of this study which is on fabricated POF splitter has been determined in order to see the performance of POF splitter at different temperatures. We can see that the POF splitter can still be used up to a temperature of $100^{\circ} \mathrm{C}$. Furthermore, its jacket has been removed and it will cause the POF splitter to be less resistant to high temperature. If the POF splitter still has a jacket that functioned as its core structure, probably it is able to accommodate with higher temperature. From this experiment, the final objective of this study which is on fabricated POF splitter has been determined in order to see the performance of POF splitter at different temperatures.

\section{CONCLUSION}

In conclusion, all of the research objectives were achieved. Seven samples of POF splitter were successfully fabricated by using low-cost fusion technique and it used simple tools such as candle and metal tubes. However based on the characterization that has been made before, performance of fabricated POF splitter is unsatisfactory. The measured power loss indicates a high in value and it shows that the fusion process is poor. But by looking at the comparison graph for POF splitter before and after polished, the loss is considerably decreased after being polished with sand study. It shows that, for each of the fusible POF splitter, cleaning process should be done carefully. For experiment on temperature, POF splitter can still be used even at high temperature up to $100^{\circ} \mathrm{C}$. The recorded loss shows that there is not really high increasing and it still can be reduced if POF splitter has special jacket of thermal insulation. However, the overall performance on this POF splitter needs to be improved in order to achieve a satisfactory level before it can be implemented in industry. Fusion technique also needs to be improved so that the POF can be fused properly. As a result, the POF splitter will be consistent in performance. This is because, the POF splitter gives different performance and it is difficult to make a characterization and conclusion.

Future work: Further study in fusion process should be done in order to improve the performance of POF splitter, so that the loss can be decrease and the efficiency will exceed $90 \%$. A device that can measure irregular pulling and twisting force will give more satisfactory performance on POF splitter. The method on heating that can provide not very high temperature 
throughout middle of POF should also be noting. In addition, a special jacket of thermal insulation for POF splitter can also be proposed to design and develop. It is because the splitter is shielded from heat and it can reduce the loss that has been occurred.

\section{REFERENCES}

Ab-Rahman, M.S., H. Guna, M.H. Harun, S.D. Zan and K. Jumari, 2009a. Cost-effective fabrication of self-made $1 \times 12$ polymer optical fiber-based optical splitters for automotive application. Am. J. Eng. Applied Sci., 2: 252-259. DOI: 10.3844/ajeassp.2009.252.259

Ab-Rahman, M.S., H. Guna and M.H. Harun, 2009b. Cost-effective $1 \times 12$ POF-based optical splitters as an alternative optical transmission media for multipurpose application. Int. J. Comput. Sci. Network Security, 9: 72-78.

Ab-Rahman, M.S., H. Guna, M.H. Harun, and K. Jumari, 2010a. Fabrication and characterization of hand-made $1 \times 12$ splitter based on polymer optical fiber made of polymethyl methacrylate. Sains Malaysiana, 39: 459-466.

Ab-Rahman, M.S., H. Guna, M.H. Harun, Zan., M.S.D. Zan and K. Jumari, 2010b. Home-made optical $1 \times 12$ fused-taper-twisted polymer optical fiber splitters for small world communication. J. Applied Sci. Res., 6: 2212-2218.

Appajaiah, A., 2004. Climatic stability of Polymer Optical Fibers (POF). Ph.D. Thises, Potsdam University.
Grzemba, A., 2008. MOST: The Automotive Multimedia Network. 1st Edn., Franzis, Poing, ISBN: 3772353169, pp: 383.

Harun, M.H., M.S. Ab-Rahman, H. Guna and K. Jumari, 2011. Novel fused $1 \times \mathrm{N}$ polymer coupler for video surveillance applications. Proceedings of the IEEE Symposium on Business, Engineering and Industrial Applications, Sept. 25-28, IEEE Xplore Press, Langkawi, pp: 486-490. DOI: 10.1109/ISBEIA.2011.6088863

Im, S.H., D.J. Suh, O.O. Park, H. Cho and J.S. Choi et al., 2002. Fabrication of a graded-index polymer optical fiber preform by using a centrifugal force. Korean J. Chem. Eng., 19: 505-509. DOI: 10.1007/BF02697164

Kuzyk, M.G., 2007. Polymer Fiber Optics: Materials, Physics and Applications. 1st Edn., CRC/Taylor and Francis, Boca Raton, USA., ISBN: 1574447068, pp: 399.

O'Gorman, M., M. Scholles and J. Faller, 2010. Simply POF: High-end connectivity with plastic optical fiber. Fraunhofer Institut Photonische Mikrosysteme, Dresden.

Rahman, M.S.A., L.S. Supian, H. Guna, M.H. Harun and K. Jumari, 2012. Performance of thin-film as wavelength filter in WDM-POF network. Am. J. Applied Sci., 180-185. DOI: 10.3844/ajassp.2012.180.185

Zieamann, O., J. Krauser, P.E. Zamzow and W. Daum, 2008. POF Handbook: Optical Short Range Transmission Systems. 2nd Edn., Springer, Berlin, ISBN: 3540766286, pp: 880. 